\title{
2) Some Experimental Methods in Biorheology
}

\author{
Eiichi FuKADA
}

The Institute of Physical and Chemical Research, Yamato, Saitama

Viscosity and elasticity are the most fundamental quantities in the rheological properties of biological materials. Three noble apparatuses for measuring the steady flow viscosity and the dynamic viscoelasticity are described in this paper.

The first is a new type of capillary viscometer, which needs only $1 \mathrm{ml}$. of the liquid specimen. Two syringe needles are jointed to the upper and lower ends of a small glass cylinder. A small metal bellows is attached to the cylinder, in order to reduce the pressure in the tube. If the lower needle is inserted into the liquid specimen, the liquid is drawn into the tube through the capillary of the needle. The air is at the same time drawn into the tube through the capillary of the upper needle. Since the dimension of the needles and the viscosity of air are known, the height of the liquid drawn in directly measures the viscosity of the liquid. The viscosity of blood taken from about 200 patients was examined by this viscometer and was compared with the diagnosis of the disease. No definite correlation between them was found except for the case of anemia. The shear rate used in the viscometer is about $10^{3} \mathrm{sec}^{-1}$, where the rouleau of red blood cells is already disintegrated by the rapid flow of the blood. The viscosity of blood must be measured at the much lower rate of shear in which the blood cells are agglutinated to form rouleaus.

The second apparatus is a rotational viscometer available at a wide range of shear rate. Two concentric cylinders with an annular gap of $1 \mathrm{~mm}$. and a height of about $5 \mathrm{~cm}$. are placed in a water thermostat. About $5 \mathrm{ml}$. of the blood is introduced into the gap. The outer tube is rotated by a motor and a gear in variable speeds, covering the shear rate from 0.02 to $80 \mathrm{sec}^{-1}$. The torque given to the inner tube due to the viscosity of the blood is detected by an device similar to the electric balance and led to the vertical axis of an XY recorder. The speed of rotation of the outer tube is also electrically detected and introduced to the horizontal axis of the $X Y$ recorder. The flow curve i. e., the stress vs. shear rate is thus recorded on a graph paper in about $30 \mathrm{sec}$. From the curve, the steady flow viscosity is easily obtained as a function of shear rate.

The variation of the viscosity with shear rate (non-Newtonian viscosity) was not observed for plasma. In blood, however, the viscosity gradually decreased with the increase of shear rate, indicating a typical non-Newtonian viscosity. If the shear rate first increases from zero, the aggregates of red blood cells (rouleau) tend to be disintegrated. If the shear rate is further increased, the individual cells are deformed so as to minimize the resistance to flow of the blood. These two mechanisms are 
usually proposed to explain the gradual decrease of the viscosity of blood with the shear rate.

The last apparatus has been designed for measuring the dynamic viscoelasticity of blood during clotting and of the biological tissues such as blood vessel walls. The blood is filled into an annular gap of $1 \mathrm{~mm}$. width between two concentric cylinders with the diameters of about $10 \mathrm{~mm}$. and the height of about $30 \mathrm{~mm}$. The outer cylinder is given a vertical sinusoidal oscillation at $10 \mathrm{c}$. $/ \mathrm{sec}$., with a fixed amplitude, which is measured by a strain gauge. The oscillating stress applied to the inner cylinder due to the viscoelasticity of clotting blood is detected by another strain gauge. The electrical signal of the stress is amplified and electrically divided into two components; one of the same phase with the strain, the other being 90 degree out of phase with the strain. The former gives the dynamic elastic modulus and the latter the loss modulus, which is the product of the dynamic viscosity and the angular frequency. Thus the automatic recording of the time variation of dynamic modulus and loss modulus of blood is pessible during its entire range of clotting. The process of gellation of fibrin fibers incorporated with the capture of red blood cells was quantitatively investigated by this apparatus. 